\title{
Aplicación del software Matlab, como estrategia metodológica en la enseñanza-aprendizaje de cálculo de una variable a nivel superior de ingeniería de telecomunicaciones de la UTA.
}

\begin{abstract}
Aplicación del software Matlab, como estrategia metodológica en la enseñanza-aprendizaje de Cálculo de una Variable a nivel superior de Ingeniería de Telecomunicaciones de la UTA"
\end{abstract}

Jaime Rodrigo Guilcapi Mosquera. ${ }^{1}$, Juan Manuel Martínez Nogales. ${ }^{2}$,Myrian Cecilia Borja Saavedra. ${ }^{3}$ Libio Enrique Guilcapi Mosquera. ${ }^{4}$

\begin{abstract}
.
The evaluation of the process of teaching Matlab to students was a carried out, as a methodology for learning the calculation of a variable at a higher level. I determine that his taste for the subject as it increased creativity and exploitation logical creative reasoning. The purpose of this paper is to make known the influence of Matlab's training as a teachinglearning instrument for calculating a variable at the higher level of Telecommunications Engineering at UTA. It is a based on a descriptive and quasi-experimental study
\end{abstract}

Keywords: Matlab, Methodological Strategy, teaching-learning, calculation of a variable, Telecommunications Engineering

\footnotetext{
${ }^{1}$ Universidad Técnica de Ambato, Ingeniería en Telecomunicaciones, Ambato, Ecuador, jr.guilcapi@uta.edu.ec ${ }^{2}$ Escuela Superior Politécnica de Chimborazo, Facultad de Recursos Naturales, Carrera de Ingeniería

Forestal. Riobamba, Ecuador. jumartinez@espoch.edu.ec

${ }^{3}$ Escuela Superior Politécnica de Chimborazo, Facultad de Ciencias, Riobamba, Ecuador.mc_borja@yahoo.es

${ }^{4}$ Pontifica Universidad Católica, Sede Ambato, Ambato, Ecuador. Iguilcapim@ gmail.com
} 


\section{Resumen.}

Se realizó la evaluación del proceso de la enseñanza con Matlab a los estudiantes, como metodología en el aprendizaje del cálculo de una variable a nivel superior. Determino que su gusto por la asignatura ya que aumento la creatividad y la concertación del razonamiento lógico creativo. Este escrito tiene como fin dar a conocer la influencia de la capacitación del Matlab como instrumento de enseñanza- aprendizaje del cálculo de una variable a nivel superior de la Ingeniería en Telecomunicaciones. Se fundamenta en un estudio descriptivo y cuasi experimental. La comprobación de resultados del Pre y Post test se empleó la prueba no paramétrica McNemar, Chi Cuadrado entre otras que dan el verdadero sustento a esta investigación.

Palabras claves: Matlab, Estrategia Metodológica, enseñanza-aprendizaje, cálculo de una variable, Ingeniería Telecomunicaciones

\section{Introducción.}

Las Tecnologías de la Información y la Comunicación (TICS) han tenido un desarrollo explosivo en la última parte del siglo XX y el comienzo del siglo XXI, al punto de que han dado forma a lo que se denomina "Sociedad del Conocimiento" o "de la Información". Prácticamente no hay un solo ámbito de la vida humana que no se haya visto impactada por este desarrollo: la salud, las finanzas, los mercados laborales, las comunicaciones, el gobierno, la productividad industrial, etc. El conocimiento se multiplica más rápido que nunca antes y se distribuye de manera prácticamente instantánea. El mundo se ha vuelto un lugar más pequeño e interconectado. Para bien y para mal, las buenas y las malas noticias llegan antes: los hallazgos de la ciencia, nuevos remedios y soluciones, descubrimientos e innovaciones, pero también las crisis económicas, las infecciones, nuevas armas y formas de control. (UNESCO, 2013, p.10)

Las TICS se han incorporado a pasos agigantados en las instituciones de educación superior, lo que ha permitido insertarlas al mundo global, facilitando la interactividad entre las personas e instituciones a nivel mundial eliminando barreras de espacio y tiempo. Se evidencia posiciones activas ante el reto de ponerse al día con las tecnologías y el establecimiento de nuevos paradigmas educativos (Gil, Morales y Basantes,2014; Tedesco,2017; Jiménez y Fiqueroa,2017)

En nuestro país varias instituciones educativas de nivel superior (IES), poseen dificultades para gestionar un aprendizaje que conduzca al estudiante desde donde se encuentra hacia donde se desea que este (Peñaherrera, 2016).

En cuanto al uso de la tecnología, los estudiantes expresan que un grupo de docentes aproximadamente un $30 \%$, no hace usos de ella pese a las aulas cuentan con tecnología y el internet está abierta. Esto concuerda con el estudio realizado por Larrea (2013), señalando que existe debilidades en el uso y aplicación de las Tecnologías de la Información y las Comunicaciones (TICS) en los procesos de educación superior, por lo que, en la mayoría de los caos, el aula es el único medio metodológico para definir la construcción del conocimiento.

Una clasificación muy buena de los MECs es la propuesta por Thomas Dwyer (1974) en la actualidad es de mayor difusión, ya que se encuentra ligada al enfoque educativo que predomina en ellos: algoritmo o heurístico. El tipo algorítmico se visualiza por el predominio del aprendizaje vía transmisión de conocimiento, desde quien sabe hacia quién lo desea aprender y donde el diseñador se encarga de encapsular secuencias bien diseñadas de 
actividades de aprendizaje que conducen al aprendiz desde donde está hasta donde desea llegar de manera secuencial con actividades bien diseñadas, heurístico (el estudiante mediante la experiencia debe llegar al conocimiento, creando sus propios modelos de pensamiento) ( Panqueva ,1988).

- Para la implementación del Matlab, se partió de una revisión previa de la guía elaborada y de la fundamentación matemática requerida para la resolución de los problemas que se plantean. A continuación, se procedió a la revisión de las herramientas que contiene el software y la aplicación con problemas teóricos básicos, lo que permitía posteriormente utilizar esta herramienta para resolver problemas propios de la temática abordada en la asignatura, principalmente la aplicación a problemas que se deban abordar por medio del cálculo de una variable con condiciones de integración. Que son temas importantes en el manejo de la Carrera de Telecomunicaciones de la (Universidad Técnica de Ambato) que promueve al estudiante a desarrollar su capacidad creativa y la consolidación del razonamiento lógico, potenciando de esta manera, su habilitación como seres autónomos, creativos, críticos y emprendedores con capacidades y competencias para desenvolverse en un contexto cada vez más variable e incierto.

- Por otro lado, el gusto de las matemáticas se ha agudizado tanto a nivel secundario como superior, no hay maestros expertos en esta materia, por lo que las matemáticas van siendo ocupadas por profesionales que si bien sabe resolver problemas de matemáticas no demuestran su importancia creativa desapareciendo así el razonamiento del porqué de cada elemento abstracto, es decir la esencia de la matemática.

Este problema debe ser enfrentado por las Universidades preparando docentes especialistas de estas asignaturas para que en un tiempo muy cercano tengamos estudiantes convencidos de la investigación, al problema no se lo enfrenta en forma directa y desde sus raíces, (la enseñanza) seguirán saliendo de los colegios estudiantes desinteresados por la Matemática o estudiantes que piensen que los problemas matemáticos se resuelven de manera mecánica. Si seguimos con esta mentalidad la sociedad continuará rezagada del avance tecnológico, porque no vamos a tener gente preparada para enfrentar los problemas con razonamiento.

Algunos factores o variables pueden ser: El desinterés de los estudiantes en el uso adecuado de las herramientas tecnológicas, así como los problemas psicológicos, emocionales o de salud, económicos, medio social en el cuál se desenvuelven entre otras., todo esto afecta al estudiante en el proceso o nivel de enseñanza aprendizaje de las matemáticas, el problema es tan grande por lo que no se lo podrá controlar totalmente, pero si motiva en la mayoría la utilización de software Matlab para la resolución de cálculo de una variable como herramienta de comprobación de los procesos lógicos razonativos e incluso creativo, que serán tomados en cuenta cuando se realicen las evaluaciones de los contenidos.

El objetivo de este artículo es dar a conocer la influencia de la utilización del Matlab en la enseñanza aprendizaje, para su aplicación en la docencia, como metodología en la enseñanza del cálculo de una variable a nivel de pregrado. 


\section{Metodologia.}

Para la demostración de la relación existente entre las dos variables planteadas: el Plan de Capacitación en el manejo de Matlab y el aprendizaje del cálculo de una variable, fue necesario aplicar una lógica descriptiva y cuasi-experimental en una investigación que recoge los criterios y puntos de vista de los actores principales de la academia dentro del contexto universitario en estudio.

El grupo al que fue aplicado el experimento fue seleccionado de forma puntual y dirigida. Además, se consideraron aspectos de afinidad y facilidades del conocimiento en las Ciencias de Dinámica y áreas bajo la curva, de acuerdo a una optimización de recursos y tiempo. Para el estudio se utilizaron tres variables: disponibilidad, frecuencia de uso, y uso adecuado (Tamayo, 2004; Gómez \& Roquet, 2012).

Se consideró en la variable Disponibilidad a todos los elementos que el docente debe disponer en un servicio de alojamiento de archivos, aula virtual, y un blog por cada asignatura. En el caso de la Frecuencia de Uso se midió la secuencia y sistematicidad con que cada docente utilizó tanto el servicio de alojamiento de archivos y su aula virtual, como su blog. Se consideró para este estudio una sola asignatura y un mínimo 2 horas semanales es decir se utilizó las horas PAE (1 encuentro de 2 horas). Por último, en el caso del Uso Adecuado, como indicador, se consideró como manejo adecuado cuando las herramientas mencionadas anteriormente se utilizaron de acuerdo a su propósito y facilidades de uso.

La investigación realizada se desarrolló con toda la población; es decir, el docente de primer semestre de la Carrera de Telecomunicaciones (2 docentes). Además, se involucró a 60 estudiantes en los períodos académicos (abril-agosto 2018, septiembre 2018-febrero 2019). La asignación del docente a cada grupo lo realizó de manera accidental, seleccionándoles, de esta manera, bajo criterio único del maestro de la asignatura o materia. Esto posibilitó definir un grupo de control y un grupo de tratamiento, tal y como se muestra en la tabla.

Tabla 1 Distribución de los grupos de tratamiento y de control

\begin{tabular}{lll}
\hline Grupo 1 & Grupo de Tratamiento & 2 docentes de la FISEI \\
\hline Grupo 2 & Grupo de Control & 1 docentes de Invitado \\
\hline
\end{tabular}

En el grupo de tratamiento conformado por 2 docentes de la Facultad de Ingeniería en Sistemas Electrónica e Industrial la carrera de Telecomunicaciones, se aplicó el plan de capacitación, mientras que el grupo de control conformado por el docente invitado, siguió impartiendo sus clases de una manera habitual.

Con respecto a los estudiantes se manejó al primer semestre paralelo B de la carrera de Telecomunicaciones, lo que corresponde a 60 estudiantes en total. Posteriormente, se realizó la caracterización de la situación actual de las herramientas 
tecnológicas, lenguaje Matlab, con las que cuenta la institución para el desempeño académico de los docentes, de acuerdo a la información obtenida en la institución (Tabla 2).

Tabla 2 Lenguaje Matlab para uso del docente en las aulas

\begin{tabular}{ll}
\hline HERRAMIENTA & PROVEEDOR \\
\hline Sistema de Gestión de Aprendizaje (Aulas Virtuales) & MOODLE \\
Blogs & Blogger \\
Servicio de Alojamiento de Archivos & OneDrive \\
\hline Fuente: CTE &
\end{tabular}
Fuente: CTE

El método científico fue útil para analizar los resultados de la medición de las tres variables estudiadas (disponibilidad, frecuencia de uso y uso adecuado de Matlab). La técnica utilizada fue la encuesta. Esta permitió recopilar información relevante. Para la recolección de información, el instrumento empleado fue el cuestionario en el cual se incluyeron: datos generales del estudiante y docente, importancia otorgada, conocimiento sobre Matlab, disponibilidad, frecuencia de uso, y uso adecuado del mismo.

La encuesta Pre se aplicó a toda la muestra, y se realizó a los docentes seleccionados en los diferentes cursos que reciben Física y Calculo de una variable de la carrera de Telecomunicaciones de la FISEI la Universidad Técnica de Ambato.

Una vez recolectada la información, se tabularon los datos y se obtuvieron los resultados del estudio Pre. Se desarrolló el plan de capacitación orientado al uso adecuado del Matlab, para posteriormente ser aplicado en el Grupo de Tratamiento. Posteriormente se aplicó el plan de Capacitación y se procedió a la preparación del cuestionario de estudio Post, en donde se seleccionaron las mismas preguntas del aspecto uso del Matlab realizadas en el estudio Pre. La encuesta Post se aplicó al grupo de Tratamiento; es decir, a los 3 docentes de la carrera de Telecomunicaciones de la FISEI y los estudiantes. Recolectada la información se tabularon los datos y se obtuvieron los resultados del estudio Post. Finalmente, se realizó el análisis estadístico de los resultados obtenidos entre el grupo de estudio y el grupo de control.

Para la tabulación de información, procesamiento de datos y la comprobación de la hipótesis, se utilizó las herramientas informáticas EXCEL y MINITAB, permitiendo visualizar los resultados en tablas y gráficos estadísticos para su análisis e interpretación correspondiente. Se emplearon las frecuencias absolutas y porcentajes para las variables cualitativas. Para la comparación de los resultados del Pre y Post test se empleó la prueba no paramétrica McNemar. Para las variables cuantitativas se emplearon como medidas resumen las de tendencia central (media) y medidas de dispersión (desviación estándar). A fin de realizar la comparación de promedios de notas se realizó la prueba paramétrica Comparación de medias para muestras independientes, previa comprobación de la normalidad de las observaciones a través de la prueba de bondad de ajuste Kolmogorov-Smirnov.Con el propósito de comprobar la homocedasticidad de varianzas, se realizó la prueba de Levene. 
Para todas las pruebas de hipótesis empleadas se utilizó un nivel de significación del 5\%, y se obtuvieron los resultados que se presentan a continuación.

En la tabla 3 se muestran los resultados de la evaluación del Pre test en cuanto al uso de las TICS por los docentes. Puede verse, que, de los 160 docentes, la mitad (50\%) poseen blogs; de ellos, el 45\% (36 docentes) lo usaron al menos dos veces por semana, y solo el 30\% (24 docentes) lo usa adecuadamente. Por otra parte, un 60\% (96 docentes) poseen aulas virtuales; de ellos, el $51 \%$ (49 docentes) la usaron al menos dos veces por semana y solo un $24 \%$ ( 23 docentes) la usó adecuadamente. En relación con el servicio de alojamiento de archivos, el $90 \%$ (144 docentes) utilizaron un servicio de alojamiento de archivos; de ellos, menos del $50 \%$ (el $43 \%$, que corresponde a 62 docentes) lo usaron al menos dos veces por semana, y más del 50\% (el 64\%, que correspondió a 92 docentes) lo usaron adecuadamente.

Tabla 3 . Evaluación Pre del uso de las TICS por los docentes de la A NIVEL SUPERIOR

\begin{tabular}{|c|c|c|c|c|c|c|c|c|c|c|c|c|}
\hline & \multicolumn{4}{|c|}{ DISPONIBILIDAD } & \multicolumn{4}{|c|}{ FRECUENCIA } & \multicolumn{4}{|c|}{ USO ADECUADO } \\
\hline & \multicolumn{2}{|c|}{ SI } & \multicolumn{2}{|c|}{ NO } & \multicolumn{2}{|c|}{ SI } & \multicolumn{2}{|l|}{ NO } & \multicolumn{2}{|c|}{ SI } & \multicolumn{2}{|c|}{ NO } \\
\hline & $\#$ & $\%$ & $\#$ & $\%$ & $\#$ & $\%$ & $\#$ & $\%$ & \# & $\%$ & $\#$ & $\%$ \\
\hline $\mathrm{B} \log s$ & 80 & 50 & 80 & 50 & 36 & 45 & 44 & 55 & 24 & 30 & 56 & 70 \\
\hline $\begin{array}{c}\text { Aulas } \\
\text { virtuales }\end{array}$ & 96 & 60 & 64 & 40 & 49 & 51 & 47 & 49 & 23 & 24 & 73 & 76 \\
\hline $\begin{array}{l}\text { Servicio de } \\
\text { alojamiento } \\
\text { De archivos }\end{array}$ & 144 & 90 & 16 & 10 & 62 & 43 & 82 & 57 & 92 & 64 & 52 & 36 \\
\hline
\end{tabular}

Al realizar el Post test puede apreciarse, en la tabla 4, que más del 60\% (67\%, 107 docentes) poseían blogs; de ellos, más del 50\% (53\%, 57 docentes) lo usaron al menos dos veces por semana y el 43\% (46 docentes) lo usó adecuadamente. Hubo un 77\% (123 docentes) que poseían aulas virtuales; de ellos, el mayor por ciento (65\%, 80 docentes) correspondió a docentes que las utilizaron al menos dos veces por semana y un 85\% (105 docentes) las emplearon adecuadamente. Por otra parte, el 95\% (152 docentes) utilizó un servicio de alojamiento de archivos; de ellos, un $43 \%$ (65 docentes) lo utilizaron al menos dos veces por semana y un $75 \%$ (114 docentes) lo utilizaron adecuadamente

Tabla 4. Resultados de la evaluación Post test del uso de las TICS por los docentes

\begin{tabular}{|c|c|c|c|c|c|c|c|c|c|c|c|c|}
\hline & \multicolumn{4}{|c|}{ DISPONIBILIDAD } & \multicolumn{4}{|c|}{ FRECUENCIA } & \multicolumn{4}{|c|}{ USO ADECUADO } \\
\hline & \multicolumn{2}{|c|}{ SI } & \multicolumn{2}{|c|}{ NO } & \multicolumn{2}{|c|}{ SI } & \multicolumn{2}{|l|}{ NO } & \multicolumn{2}{|c|}{ SI } & \multicolumn{2}{|c|}{ NO } \\
\hline & \# & $\%$ & \# & $\%$ & $\#$ & $\%$ & \# & $\%$ & \# & $\%$ & \# & $\%$ \\
\hline $\mathrm{B} \log \mathrm{s}$ & 107 & 67 & 53 & 33 & 57 & 53 & 50 & 47 & 46 & 43 & 61 & 57 \\
\hline Aulas virtuales & 123 & 77 & 37 & 23 & 80 & 65 & 43 & 35 & 105 & 85 & 18 & 15 \\
\hline $\begin{array}{c}\text { Servicio de alojamiento } \\
\text { De archivos }\end{array}$ & 152 & 95 & 8 & 5 & 65 & 43 & 87 & 57 & 114 & 75 & 38 & 25 \\
\hline
\end{tabular}


La tabla 5 muestra los resultados de la prueba no paramétrica McNemar para muestras pareadas. Puede verse, que en todos los aspectos esta prueba fue estadísticamente significativa $(\mathrm{p}<0.05)$ excepto para la Frecuencia de uso en el Servicio de alojamiento de archivos. Esto significa que existió un cambio en el uso de las TICS por los docentes, después de aplicado el Plan de Capacitación en el manejo de Matlab y este cambio fue para mejora de los docentes

Tabla 5 Comparación de resultados del Pre y Post test del uso de las TICS por los docentes.

\begin{tabular}{|c|c|c|c|}
\hline \multicolumn{2}{|c|}{ ASPECTOS } & $\begin{array}{c}\text { ESTADISTICO } \\
\text { DE } \\
\text { CONTRASTE }\end{array}$ & $\begin{array}{l}\text { VALOR } \\
\mathrm{P}\end{array}$ \\
\hline \multirow{4}{*}{ DISPONIBILIDAD } & $\mathrm{B} \log \mathrm{s}$ & 250.372 & $0.000^{*}$ \\
\hline & Aulas Virtuales & 250.372 & $0.000^{*}$ \\
\hline & $\begin{array}{c}\text { Servicio de alojamiento de } \\
\text { archivos }\end{array}$ & - & $0.008^{*}$ \\
\hline & $\mathrm{B} \log \mathrm{s}$ & - & $0.000^{*}$ \\
\hline \multirow{3}{*}{$\begin{array}{c}\text { FRECUENCIA DE } \\
\text { USO }\end{array}$} & Aulas Virtuales & 290.372 & $0.000^{*}$ \\
\hline & $\begin{array}{c}\text { Servicio de alojamiento de } \\
\text { archivos }\end{array}$ & - & 0.250 \\
\hline & Blogs & - & $0.000^{*}$ \\
\hline \multirow[b]{2}{*}{ Uso adecuado } & Aulas Virtuales & 71.014 & $0.000^{*}$ \\
\hline & $\begin{array}{l}\text { Servicio de alojamiento de } \\
\text { archivos }\end{array}$ & - & $0.000^{*}$ \\
\hline
\end{tabular}

Nota: 1: Prueba de McNemar, 2: Chi-cuadrado Corregido por continuidad, *: p $<0.05$

\section{Resultados.}

La media de las notas para el grupo control fue de 16.00 puntos con una desviación estándar de aproximadamente 2,67 puntos, en tanto para el grupo experimental la media fue de 16,58 puntos con una desviación estándar de aproximadamente 3 puntos. Esto significa que ambos grupos tuvieron puntuaciones medias muy similares. En el gráfico 1, se observa cómo los intervalos de confianza de ambos grupos se solapan. Esto nos da una idea de que puede no existir diferencia entre las medias de ellos. 
Gráfico 1 Intervalos de confianza de las medias de los grupos Control y Experimental.

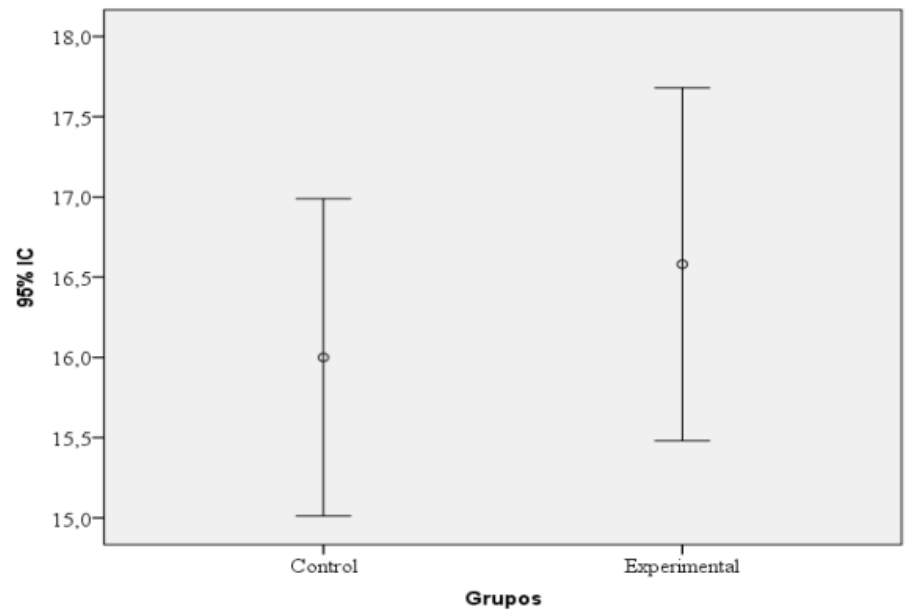

En la tabla 6, se observan los resultados de la Prueba de comparación de medias para muestras independientes, donde se compararon las medias del Grupo Control y las del Grupo Experimental. Previo a la realización de esa prueba paramétrica, se realizó la comprobación de la normalidad a través de la prueba no paramétrica Kolmogorov-Smirnov, que fue estadísticamente no significativa, con lo que no se rechazó la normalidad ( $p=0,200)$. Se procedió entonces a realizar la Prueba de Levene, con el fin de comprobar la homocedasticidad de varianzas $(\mathrm{F}=0,210, \mathrm{p}=0,648$ 0,648), con lo se concluyó que existe homogeneidad de varianzas y se pudo realizar la prueba $\mathrm{T}$ para igualdad de medias. La prueba resultó no significativa $(\mathrm{t}=-0,802, \mathrm{gl}=60, \mathrm{p}=0,426)$, siendo la diferencia de medias de -0.581 y un intervalo de confianza para la diferencia de medias de $-2,029 ; 0,868$

Tabla 6 Resultados de la prueba Comparación de medias entre los grupos Control y Experimental

\begin{tabular}{|c|c|c|c|c|c|c|c|c|}
\hline & \multicolumn{2}{|c|}{$\begin{array}{c}\text { Prueba de } \\
\text { Levene } \\
\text { Para la } \\
\text { igualdad de } \\
\text { varianza }\end{array}$} & \multirow{3}{*}{$\mathbf{t}$} & \multicolumn{5}{|c|}{ Prueba $T$ para la igualdad de medias } \\
\hline & $\mathbf{T}$ & $S_{i}$ & & \multirow{2}{*}{ gl } & \multirow{2}{*}{$\begin{array}{c}\text { Valor } \\
\mathbf{p}\end{array}$} & \multirow{2}{*}{$\begin{array}{l}\text { Diferencia } \\
\text { de medias }\end{array}$} & \multicolumn{2}{|c|}{$\begin{array}{l}95 \% \text { de confianza } \\
\text { para la diferencia }\end{array}$} \\
\hline & $\mathrm{F}$ & Sig & & & & & $\begin{array}{l}\text { Límite } \\
\text { Inferior }\end{array}$ & $\begin{array}{c}\text { Limite } \\
\text { Superior }\end{array}$ \\
\hline $\begin{array}{l}\text { Fundamentamos } \\
\text { con varianzas } \\
\text { iguales. }\end{array}$ & 0.210 & 0.648 & $\overline{-}-802$ & 60 & 0.426 & -0.581 & -2.029 & 0.868 \\
\hline
\end{tabular}


Con estos resultados anteriores puede plantearse que los dos grupos tuvieron un comportamiento similar. Las notas de ambos grupos fueron muy similares. Se sugiere en futuras investigaciones incrementar el tamaño muestral, a partir de estudios interuniversitarios, que incluyan docentes y estudiantes de otras FISEI del país, en las cuales se aplique la herramienta Matlab para la comprobación de los procesos creativos y lógicos de razonamiento.

\section{Conclusiones.}

- La consideración de los actores intervinientes, las formas de organización institucional adoptadas La consideración de los actores intervinientes, las formas de organización institucional adoptadas aprendizaje, es hoy una exigencia consustancial a la educación superior.

- Como resultado de la investigación realizada se consiguió la caracterización de la situación actual de las herramientas tecnológicas con lenguaje Matlab con las que cuenta la institución. El análisis de los criterios y parámetros del uso adecuado del Matlab, en la docencia a nivel superior, por parte de los docentes y estudiantes en el proceso de enseñanza de cálculo de una variable aplicadas en problemas de ingeniería de la UTA, posibilita mejorar las alternativas de enseñanza - aprendizaje Finalmente, la comprobación de la capacitación del uso de Matlab como alternativa para solucionar problemas de ingeniería, mediante el uso de herramientas estadísticas por el conjunto amplio de asignaturas como Física y Resistencia de circuitos promueven el interés del estudiante en el contexto de cada carrera de la FISEI.

\section{Referencias bibliográficas.}

- Asamblea Nacional del Ecuador. (2008). Constitución de la Republica de Quito, Ecuador. Recuperador de:

- http://www.asambleanacional.gob.ec/es/contenido/constitucion-de-la-republicadel-ecuador

- CEACES. (2015a). Resolución No. 130-CEACES-SE17-201.Formato autoevaluación IES, Quito, Ecuador.

- CEACES. (2015b). Modelo Genérico de Evaluación del entorno de aprendizaje de carreras presenciales y semipresenciales de las universidades y escuelas politécnicas del Ecuador, Quito, Ecuador.

- Dwyer. T.A. (1974). Heuiristic strategies for using computers to enrich education. International Journal of ManMachine Studies, 6 (2), 137-154.

- Gil Álvarez, J- L, Morales Cruz, M. y Basantes Garcés, J. L. (2014). Una aproximación a la calidad universitaria a partir de los procesos de evaluación y acreditación. Universidad y Sociedad, 7 (1), 17-21. Recuperado de: http://rus.ucf.edu.cu/

- Gómez, D. R \& Roque, J. V. (20125). Metodología de la investigación. México, Tlalnepantla: Red Tercer Milenio. 
- Jiménez, A. y Figueroa, H. (2017). Políticas educativas en la universidad superior: 1952-1922. Revista Colombiana de Educación, (38-39). Recuperado de: http://revistas-pedagocia.edu.co/indez.php/RCE/article/view/5439/4466

- Larrea Granados, E. (2013). El currículo de la educación superior desde la complejidad sistemática. Ecuador, Quito: CES.

- Panqueva, A. H. G. (1988). Ambiente de enseñanza-aprendizaje enriquecidos con computador. Boletín de Informática Educativa, 1(2), 117-139.

- Peñaherrera, M. (2016). Evaluación de un programa de fortalecimiento del aprendizaje basado en el uso de las TIC en el contexto ecuatoriano. Revista iberoamericana de Evaluación Educativa,4(2).

- SENDPLADES. (2013). Plan Nacional para el Buen Vivir 2013-2017. Quito, Ecuador.

- Tamayo, M. (2004). EL proceso de la investigación científica. México, Ciudad de México: Editorial Limusa.

- Tedesco, J. C. (2017). Estrategias de desarrollo y educación: el desafío de la gestión pública. Revista colombiana de educación [S.I], (24). Recuperado de: http://revistas.um.es/red/article/view/245231/185771

- Dorfsmani, M. (2015). La Profesión docente en contextos de cambio: el docente global en la sociedad de la información. Revista de Educación a Distancia, (6). Recuperado de: http://revistas.um.es/article/view/245231/185771

- Fernandez, E. M. (2016). Aprendizaje constructivista para el análisis de estructuras mediante el uso de un entorno virtual/Constructivist learning for the análisis of structures by using a virtual enviroment. Revista Tecnociemtífica URU, (9), 41-50:

- Majó, j. y Marqués. P. (2002. La revolución educativa de la era de Internet, Colección Compromiso con la educación. España, Barcelona: Cisspraxis.

- Requema, B. E. S (2015). Las TIC y la educación social en el siglo XXI. EDMETIC, $5(1), 8-24$.

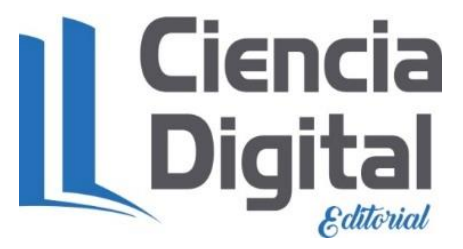




\section{Para citar el artículo indexado.}

Guilcapi Mosquera, J., Martínez Nogales, J., Borja Saavedra, M., \& Guilcapi Mosquera, L. (2019). Aplicación del software Matlab, como estrategia metodológica en la enseñanzaaprendizaje de cálculo de una variable a nivel superior de ingeniería de telecomunicaciones $\begin{array}{llll}\text { de la UTA. } & \text { Explorador } & \text { 3igital, } & 3.1),\end{array}$ https://doi.org/10.33262/exploradordigital.v3i3.1.863

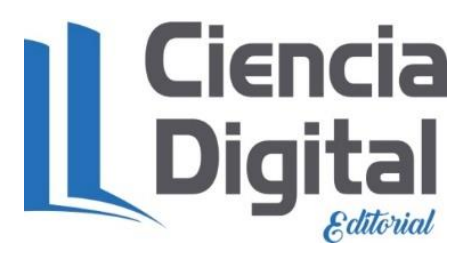

El artículo que se publica es de exclusiva responsabilidad de los autores y no necesariamente reflejan el pensamiento de la Revista Explorador Digital.

El articulo queda en propiedad de la revista y, por tanto, su publicación parcial y/o total en otro medio tiene que ser autorizado por el director o editor de la Revista Explorador

\section{Digital.}
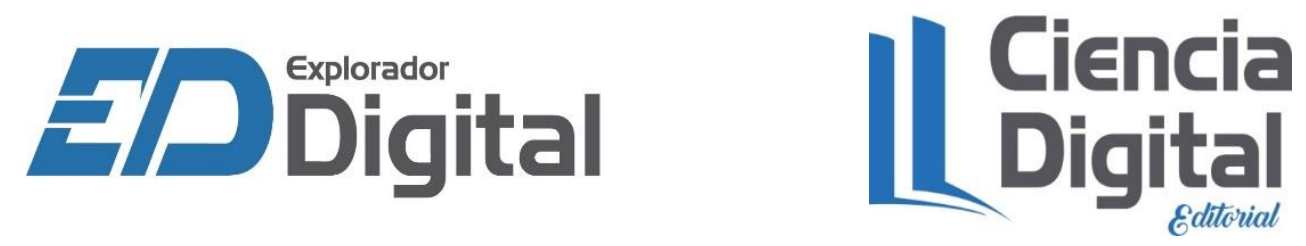